\title{
Germanica
}

\section{Portrait de femme en céleste dragon}

Les images de Marlen Haushofer dans ses récits et romans

Porträt einer Frau als himmlischer Drache. Die Bilder Marlen Haushofers in den

Erzählungen und Romanen

\section{Patrick Charbonneau}

\section{OpenEdition}

\section{Journals}

Édition électronique

URL : http://journals.openedition.org/germanica/2625

DOI : 10.4000/germanica.2625

ISSN : 2107-0784

\section{Éditeur}

Université de Lille

\section{Édition imprimée}

Date de publication : 31 décembre 1989

Pagination : 55-81

ISSN : 0984-2632

\section{Référence électronique}

Patrick Charbonneau, "Portrait de femme en céleste dragon », Germanica [En ligne], 5 | 1989, mis en ligne le 09 février 2015, consulté le 06 octobre 2020. URL : http://journals.openedition.org/germanica/ 2625 ; DOI : https://doi.org/10.4000/germanica.2625

Ce document a été généré automatiquement le 6 octobre 2020.

(c) Tous droits réservés 


\title{
Portrait de femme en céleste dragon
}

\author{
Les images de Marlen Haushofer dans ses récits et romans \\ Porträt einer Frau als himmlischer Drache. Die Bilder Marlen Haushofers in den \\ Erzählungen und Romanen
}

Patrick Charbonneau

... wenn er erschöpft eine Reise beendet hat, zu

Hause die schlimmen, auf ihre Ursachen hin nicht

mehr zu durchschauenden Folgen am eigenen

Leibe zu spüren bekommt.

Franz Kafka, Die Verwandlung.

Das Erkennen? Wirklich nur das Erkennen! - Das

Verzeihen. Das Verzeihen wovon? - Die Liebe.

Mein Gott: die Liebe.

Rainer Maria Rilke,

Die Aufzeichnungen des Malte Laurids

Brigge.

1 Dans les dernières pages de Die Mansarde, ultime roman rédigé par Marlen Haushofer sur son lit de mort, la narratrice note dans son journal intime :

Le monde est un labyrinthe dans lequel je ne retrouverai jamais mon chemin puisque ma tête ne peut travailler que sur des images et qu'elle est incapable de pensées raisonnables ${ }^{1}$.

2 La romancière autrichienne, née en 1920 à Frauenstein (Haute-Autriche) et emportée par la maladie alors qu'elle avait à peine cinquante ans, aurait-elle sombré dans l'irrationnel ? La lecture des récits et romans révèle plutôt une femme d'une sensibilité extrême, apte à saisir avec précision la psychologie des êtres qui l'entourent, sa famille et le milieu de la petite ville de province, Steyr, où elle a passé presque toute son existence; une femme à l'écoute de son époque et des grandes interrogations qui l'agitent, vivant dans sa chair et dans son âme les conflits d'une humanité en proie à la guerre, aux fascismes, aux secousses de profondes mutations technologiques, sociales, politiques; une femme entrant en résonance avec un monde divisé et chaotique dont 
elle cherche, par le biais de son introspection, à découvrir les mobiles et le sens caché. Nous montrerons ici que les images fortes de l'œuvre - allégories, métaphores, mythes fantasmatiques - loin d'être les élucubrations d'un esprit déréglé, s'organisent avec grande cohérence, conduisent à la « connaissance ", et à l'élaboration d'une « religion » qui réconcilie avec soi, avec la mort, avec la Création, dans une quête dont l'objet a pour nom Amour universel.

3 Le sens de l'image se manifeste dès les premiers récits publiés en 1947. Dans Ein gemütlicher Abend zu Hause ${ }^{2}$, un homme tente d'expliquer à sa jeune épouse des formules mathématiques. Elle ne comprend pas et son esprit s'évade. La voix de son mari lui rappelle le ronronnement d'un chat, ou le rugissement d'un tigre quand il s'aperçoit qu'elle ne suit plus. Einstein lui fait penser à « un être gentil qui a quelque chose d'un lapin » (97). Il n'est pas jusqu'à sa conscience vagabonde qui ne s'incarne en un « petit diable ricanant, se roulant en boule dans sa tête tandis que les yeux écarquillés et les lèvres entrouvertes elle écoutait les paroles de son mari » (99).

Ne nous y trompons pas. Le ton enjoué de l'humour tendre n'est qu'une apparence recouvrant un profond désarroi. La jeune femme se dit «en proie à un funeste dédoublement " (96); elle parle même de "symptômes de schizophrénie » (ibid.). Les personnages des romans et récits sont tous confrontés à l'impossibilité de penser rationnellement le monde et souffrent tous d'un dédoublement de la personnalité. Ainsi, l'histoire intitulée Schreckliche Treue évoque le destin d'une jeune mère qui, dans le dernier hiver de la Seconde Guerre mondiale, alors que son mari est au front, fuit l'Allemagne du Nord pour regagner son pays natal, l'Autriche. Dans le train qui l'emmène, elle échoue à se représenter sa vie future, « car cela en restait aux pensées et il n'en résultait aucune image » (192). Tout ce qu'elle perçoit, en fermant les yeux, est "un néant rouge et noir» (ibid.). Pendant qu'elle est descendue acheter quelques provisions de bouche au buffet, le convoi repart avec l'enfant. Il sera bombardé. Son fils mort, son mari disparu à la guerre, elle se remarie, redevient mère de famille, mais son sommeil reste hanté par des cauchemars dont elle se réveille en criant. Son second mari reste pour elle "un étranger ». Si elle est tout près "d'accéder à une nouvelle réalité, à la chaleur, au bonheur d'être enfin à la maison », elle ne parvient jamais «à franchir le seuil, ni à échapper à sa terrible fidélité » (106).

5 Toute l'œuvre de Marlen Haushofer est construite sur l'existence de cette dualité entre deux mondes et la nécessité, pour ne pas aliéner son moi, de rester fidèle à l'un et à l'autre. C'est une œuvre autobiographique ${ }^{4}$ où les images masquent et dévoilent, sont les pièces d'une psychanalyse qui est aussi celle de toute une génération.

La critique, dans l'ensemble, n'a pas accordé à la guerre et au nazisme la place qui leur revient $^{5}$. Ils sont pourtant, semble-t-il, à l'origine du malaise profond de Marlen Haushofer, et de sa décision d'écrire. Elle a dix-huit ans au moment de l'Anschluß, dixneuf quand elle part en Prusse orientale effectuer son "Service du travail». Elle compose ses premiers récits dès 1946. Si quelques-uns seulement ont pour thème explicite les événements de la période national-socialiste, celle-ci obsède constamment l'imaginaire de tous les personnages dont le passé, le présent et le futur menacent de n'être jamais, sur fond de neige, qu'un « néant rouge et noir ». Ainsi Entfremdung, dans le recueil Begegnung mit dem Fremden ${ }^{6}$, relate un cauchemar qui n'est pas sans rappeler 
Rhinocéros de Ionesco, avec le même passage du singulier au pluriel, la même prolifération de l'animal inquiétant comme incarnation du "sur-homme » totalitaire :

Et puis je vis le grand coq [...]. Plus grand qu'un homme, juché sur ses pattes habillées de plumes irisées, avec une crête pourpre et un bec noir, il se tenait immobile et paraissait indiciblement étrange et prestigieux (234).

7 Très vite, la situation évolue. Ce sont d'innombrables coqs qui ont envahi la ville et entraîné tous les enfants, dans leurs habits blancs de probité et de candeur, au «PalastHotel » où ils les font disparaître. Sous la « lumière blanche » (ibid.), les coqs exercent sur la spectatrice et l'adolescente qui se tient à ses côtés répulsion et surtout fascination. À leur vue, la protégée d'Hedwig se lève et applaudit, ravie : « Le grand coq était vraiment beau » (234, v. aussi 237). Les femmes qui ont succombé à la séduction des animaux prestigieux voient leurs vêtements se couvrir «d'étranges taches brunâtres qui grandissaient à vue d'œil » (235), une «calamité » dont elles essaient de se protéger en se cloîtrant dans la chambre. Mais en vain: le bois de la porte est « malade » (236), les taches brunes croissent « comme un étrange champignon mortel» (ibid.). «La ville se mourait, sous le ciel indifférent et perpétuellement bleu le monde était à l'agonie " (ibid.). Quant aux quatre voisins, représentants de la bourgeoisie traditionnelle puisque «l'un était médecin, l'autre prêtre en civil, le troisième musicien, j'avais oublié la profession du quatrième » (235), s'ils sont eux aussi inquiets, ils n'en restent pas moins passifs, attablés « devant leurs verres à moitié vides ». Le musicien et le médecin proposent à Hedwig d'oublier en prenant un tranquillisant. Le prêtre estime de son devoir de "révéler à la dame la vérité », à savoir que "les coqs apparaissent déjà dans l'Apocalypse de saint Jean » (ibid.). Impuissante, désemparée, résignée, la société a abdiqué devant une catastrophe qui prend des allures eschatologiques. Images retournées des Quatre Cavaliers de l'Apocalypse, les quatre notables sont des figures dérisoires, confrontées à un destin qui les dépasse. Quant à Hedwig, elle apparaît comme l'allégorie de la Nation vaincue :

J'étais assise là, moi, une femme aux vêtements tombant en lambeaux, tenant dans mes bras sans vigueur le dernier enfant de notre monde (237).

8 Cependant, comme tous, elle s'estime responsable de ne pas avoir agi, par aveuglement, par égoïsme, d'avoir cru pouvoir préserver un bonheur individuel qu'elle croyait à l'abri de la politique et de l'histoire :

Même moi, dit-elle, je ne suis pas libre de toute trahison ni de toute infidélité (ibid.).

Ce rêve inquiétant, elle le raconte bien après la guerre à l'homme qui lui est le plus cher, pour lui faire comprendre qu'elle était incapable d'aimer, que l'amour était impossible. Or, le malheur n'atteint pas uniquement les témoins de l'hitlérisme. Il a aussi contaminé les enfants de la génération future, et les enfants de leurs enfants. C'est l'idée exprimée dans un autre récit au titre significatif: Der Erbe ${ }^{7}$ qui mêle savamment la féerie du conte mythique et le tragique de la réalité vécue. Fräulein Röder est une vieille femme qui héberge chez elle, après 1945, un jeune couple et son enfant. Parfois elle souffre de troubles psychiques, s'alite et refuse de s'alimenter en prétendant qu'elle est responsable de la guerre (109). Au petit Andi, elle raconte qu'elle était jadis une belle reine, que son chat était un tigre, et que l'heure viendra où, fendant sa vieille enveloppe avec son long couteau, elle se libérera de la chrysalide qui la retient prisonnière pour regagner son royaume. Alors il héritera des abeilles d'or qu'elle conserve précieusement dans une cassette. Un jour, Andi retrouve la dame noire baignant dans le rouge de son sang. L'enveloppe est restée la même, le chat ne s'est pas métamorphosé en tigre. Le petit garçon n'héritera pas des abeilles d'or, mais seulement 
d'un souvenir dont il ne dira rien à personne, non plus qu'il n'évoquera la vision indélébile de la morte, noire Melissa coupable d'avoir dérogé à son devoir, de ne pas avoir tué comme chaque année son époux, de ne pas avoir supprimé le néfaste bourdon pour que la société de la ruche puisse survivre ; coupable d'avoir cru que sans véritable expiation elle ferait renaitre l'ancienne communauté harmonieuse des abeilles. Orpheline, l'humanité n'est plus qu'un assemblage anarchique d'individus isolés, voués à brève échéance à la dispersion et à la disparition. « Le monde est tout à fait horrible " (115), dira l'enfant, et pour lui, il y aura toujours « deux univers : l'univers magique de Fräulein Röder et l'univers tout à fait ordinaire, sans aucun pont pour les relier » (109).

Le fascisme ne disparaît pas avec la mort de ses responsables directs. Tous leurs enfants en sont les victimes, les héritiers, et aussi les continuateurs : Marlen Haushofer a vécu l'hitlérisme à cet âge incertain qui marque le passage du monde protégé de l'enfance à celui de la vie adulte. Elle n'ignore pas que la jeunesse n'est pas toujours idyllique. Elle sait que l'enfance peut être cruelle; il suffit pour s'en convaincre de lire Kinder ${ }^{8}$. Mais pour elle, il existe un avant et un après, nettement différenciés et sans lien entre eux, que le court texte Die Zeit ${ }^{9}$ permet de situer chronologiquement puisqu'il cite des dates. Pour la jeune fille de l'histoire, le temps a commencé au jour de son premier rendezvous, en septembre 1937. Avant, il existait un monde où «le temps était arrêté » (185 sq.). Après, « une horloge s'était mise à égrener son tic-tac, lentement d'abord, puis de plus en plus vite, s'enflant pour devenir le hurlement frénétique d'une sirène, et finalement s'accélérer au point de devenir inaudible» (197). L'avant, tout entier relégué dans le souvenir, est isolé dans une sphère où la durée est abolie. L'après est soumis à la loi d'un écoulement temporel si rapide qu'il en devient impalpable, insaisissable, "inaudible». Pour le comprendre, il va falloir le figer et le rejeter lui aussi dans un passé révolu. En septembre 1965, la femme de quarante-cinq ans procède à une seconde coupure radicale pour "se pencher sur son passé ». Le récit Die Zeit apparaît ainsi comme le prototype des romans, depuis Eine Handvoll Leben jusqu'à Die Mansarde. Se plaçant hors de l'histoire, se détachant d'elles-mêmes, les narratrices omniscientes opposent la vision du paradis de leur enfance à des pans entiers de leur vie adulte, et le sens jaillit de cette froide et lucide confrontation. Pour reprendre une image de Die Mansarde, c'est toute une existence qui, regardée à travers une vitre comme une pièce de musée, va pouvoir être soumise à un minutieux examen ${ }^{10}$.

11 Toute au bonheur d'avoir survécu, la génération des années vingt n'a plus à l'égard du fascisme qu'un vague sentiment de culpabilité. Celle-ci a franchi le seuil de l'inconscient pour devenir existentielle. Les femmes coupées en deux ignorent ce qu'elles ont dû oublier pour pouvoir vivre. Epouses, mères de famille, elles mènent dès lors une double vie, s'occupent de leur foyer et parallèlement se livrent à ce qu'elles considèrent comme des « extravagances hors des règles d'une vie bourgeoise ».

Aussi, le fil de ces nouvelles Ariane ne permet pas de sortir du labyrinthe : il risque au contraire de les ramener au Minotaure :

Tout est si embrouillé, il faudrait que je trouve le fil et que je le rembobine, mais je ne trouve pas le début, seulement la fin ${ }^{11}$.

13 Pour accéder à la pleine conscience, « certaines $\operatorname{choses}^{12}$ » - on notera l'imprécision du vocabulaire - vont devoir nécessairement emprunter les chemins détournés de la fabulation.

Dans l'œuvre de Marlen Haushofer, la fable qui se présente sous la forme la plus achevée et la plus éclairante est sans conteste le mythe du Menschenmann, dans le récit 
du même nom ${ }^{13}$. Au travers de la figure du «mâle humain ", c'est toute l'histoire de l'humanité qui est censée se résumer en quelques pages. Fils de la "Grande Mère », la Nature, le Menschenmann commence à "réfléchir ", assassine le "tyran ", invente « les idéaux ", traite sa mère de "barbare ", écrit des poèmes où il chante "l'amour ", se découvre " une âme » - et doute que la Femme en ait une -, et finit par renier sa Grande Mère : « Tu n'existes pas, hurla-t-il, tu n'es qu'une chimère de mon esprit » (229). Quant à son épouse, il la bat, avant de tuer ses enfants. Asservie, esclave, la Femme n'est plus que la mère de douleur pleurant la mort de ses petits. Cependant, furieuse, la Grande Mère Nature le fait dévorer par une " louve hirsute ", puis sème une poignée de graines qui en germant recouvriront la Terre de végétation et lui redonneront son aspect primitif, celui d'où l'être humain était absent.

Le « mâle humain », Chronos/Saturne, inventeur et Grand-Maître du Temps, s'oppose à la «femelle humaine ", Rhéa/Cybèle, image de la fécondité et de l'amour, qui veut assurer la perpétuation de l'espèce et mène une lutte désespérée pour sauvegarder l'harmonie, l'unité idyllique de l'Homme et de la Terre. Il est dénoncé ici comme la cause de tous les maux. Or il anticipe la vision qui structure le roman Die Wand ${ }^{14}$.

Rappelons-en brièvement l'argument : après une catastrophe planétaire dont l'origine reste inconnue, tous les êtres vivants ont été transformés en statues minérales. Une femme (la narratrice) a survécu dans une vallée de montagne isolée du reste du monde par un mur invisible. Ayant trouvé refuge dans un pavillon de chasse qu'elle partage avec quelques animaux, elle finira par tuer le dernier homme après que celui-ci aura massacré le chien et le taureau. Comme le récit Der Menschenmann, Die Wand décrit une situation extrême. L'heure de l'apocalypse ayant sonné, le récit récurrent se situe en dehors de l'histoire, qu'il englobe entièrement. Par ailleurs, au paroxysme du désespoir, la femme, respectueuse de la création, s'est substituée à la Grande Mère. Il semblerait donc que la « Nature » ait triomphé de la «Culture », l'Eternité du Temps; que le Bien l'ait emporté sur le Mal. Mais en éliminant l'homme, la femme parachève l'œuvre de destruction. Elle est elle-même un monstre qui fait disparaitre une moitié du Ciel. Seule elle survit, avec une chatte et une vache, trois femelles qui sans mâle ne pourront plus pro-créer.

17 Toutefois, elle était obligée de supprimer l'homme. Elle était en quelque sorte en état de légitime défense. Quand elle envisage un instant l'hypothèse qu'un spécimen masculin ait survécu, elle en arrive à la conclusion suivante : ou bien il aurait été plus fort qu'elle et l'aurait asservie ; ou bien c'eût été l'inverse, et elle l'aurait étouffé (D50, F66). C'est pourquoi vouloir remplacer une société de type patriarcal par une société de type matriarcal, en inversant les rapports de force, reviendrait à substituer un esclavage à un autre. Au milieu du monde pétrifié, la narratrice est devenue - elle emploie elle-même le terme - une Menschenfrau ${ }^{15}$. Ce que Marlen Haushofer décrit ici, c'est l'impossibilité, dans la société figée où elle vit, d'être de plain-pied avec le monde, sa nation, son entourage, sa famille, et en particulier de former ce couple complémentaire dont elle a impérativement besoin pour être heureuse. "J'étais contente qu'il soit mort», dit l'héroïne de Die Wand en parlant de sa victime, "il m'aurait été difficile de le laisser en vie ». Mais aussitôt elle ajoute : « Ou peut-être si, je ne sais pas " (D224, F277).

18 L'incertitude qui perce dans cette dernière remarque montre que loin de prôner des solutions radicales, la femme se prononce en définitive en faveur d'un moyen terme. Les personnages de Marlen Haushofer balancent sans cesse entre l'exigence de l'idéal et 
l'acceptation d'une réalité contraignante, cherchant à mettre en pratique une règle de vie qui, dans un équilibre toujours précaire, les préserve du " chaos ». Cette attitude, Annette Xanthner, dans Die Tapetentür ${ }^{16}$, la définit clairement lorsqu'elle parle des rapports que la femme entretient avec la politique: celle qui occupe des fonctions politiques est l'otage des hommes ; elle leur donne bonne conscience et leur apporte un électorat. Celle qui ne s'engage pas est incapable de les renverser. Si elle refuse de se soumettre, il lui faut " entrer dans la résistance » - in die Résistance gehen, c'est-à-dire se plier à la nécessité tout en menant une action clandestine pour conquérir l'égalité des droits, adopter une tactique de "douce violence" faite de compromis sans compromissions.

Cette ligne de conduite à mi-chemin entre la passivité résignée et la révolte ouverte, définie dès 1957, est un idéal auquel les personnages de Marlen Haushofer aspirent mais qu'ils ne réussissent pas même à réaliser dans l'étroite cellule que constitue leur couple. Le récit Eine sonderbare Liebesgeschichte ${ }^{17}$ se présente à ce titre comme un constat d'échec. Deux amis d'enfance, êtres antithétiques, elle instinctive, dominée par ses affects, lui « objectif », " rationnel », " dépourvu de tout sentiment » $(221,223,219)$ et conscient de la relativité des valeurs, partagent leur vie après un mariage raté de part et d'autre. La narration s'ouvre sur la même ambivalence que celle des autres héroïnes à l'égard de leur compagnon :

Je ne sais pas ce que je donnerais pour être enfin débarrassée de Peter. Pas un sou naturellement, je ne veux absolument pas être débarrassée de lui (217).

L'homme dont il est question a beau être " un être supérieur » (222), en apparence le contraire d'un phallocrate ordinaire ou d'un Menschenmann, la femme n'arrive à le comprendre qu'« après des années d'efforts indicibles, et encore seulement avec la tête » (223). Il reste pour elle un "étranger ", tandis que dans cette union sans amour véritable, elle risque à tout moment d'aliéner sa personnalité :

Seulement le pire, c'est qu'à présent que je pense avec ses pensées, toutes mes actions me sont par instants incompréhensibles (227).

21 Pour la "survivante », dans une société qui en définitive est restée sensiblement la même avant et après, le "bonheur simple » qu'elle aurait pu connaître avec son premier mari, être " particulièrement charmant ", « chaleureux, actif, sensuel et gai » (226) est interdit. Elle doit se contenter d'un semblant de bonheur, d'une illusion d'amour :

Nous faisons comme si nous nous aimions, mais nous savons que nous n'avons pas le choix. Nulle part au monde il n'y a de femme pour Peter ni d'homme pour moi. Nous menons une vie paisible (230).

Dans les romans, les protagonistes ne s'abandonnent pas à cette sombre résignation, au contraire. Ce ne sont pas des femmes qui démissionnent. Chacune, à sa manière, lutte pour échapper à l'étroitesse d'une existence régie par les «trois $K$ », Kinder, Küche, Kirche. Le rejet le plus radical se trouve assurément dans Eine Handvoll Leben ${ }^{18}$. La petite Elisabeth, en pension chez les sœurs, ne ressent qu'aversion pour le Christ en croix, image de la souffrance, de la laideur et de la haine (49 sqq.), qui ne rappelle en rien l'image de son père adoré. Puis devenue adulte, jeune mariée et jeune mère, elle se jette à corps perdu dans une liaison extra-conjugale, un "oubli de soi " qui lui procure l'assouvissement charnel, mais non la plénitude à laquelle elle aspire, et la ravale au rang de l'animalité inconsciente. L'autre, l'homme, lui reste "étranger ", est identifié à un grand animal de zoo prisonnier derrière les barreaux de sa cage, plein d'une sourde hébétude, avant de devenir un cheval dont la femme caresse les naseaux pour échapper 
au malaise qui l'envahit. Le salut ne peut venir de l'union de ces deux solitudes. Il faut aller plus loin. Dans une vision panthéiste, la femme embrasse l'univers entier, vaine exaltation qui ne tarde pas à retomber :

Sa soif de l'inaccessible se mua lentement en tristesse (141).

Pour rejoindre Tailleurs, pour se fondre dans le Grand Tout, Elisabeth Pfluger simule une noyade dans les flots boueux de la rivière, abandonnant époux, enfant et amant. Elle ne reviendra que bien des années plus tard, après la mort accidentelle de son ancien mari, sous l'identité de Mrs. Betty Russel, riche Américaine se dissimulant derrière ses lunettes foncées. Venant de racheter la maison familiale désormais habitée par son fils, son ancienne amie devenue la seconde Mme Pfluger et la fille de celle-ci, elle sera hébergée dans la Fremdenzimmer', chambre d'amis, mais surtout " chambre de l'étrangère ", où elle dressera le constat de son passé en feuilletant un vieil album de photographies. Le lecteur n'apprendra rien du second destin d'Elisabeth-Betty. Il ne saura pas vers quel exil elle était partie pour conquérir son inaccessible royaume. Mais ce premier roman par trop "romanesque» se termine néanmoins sur une note optimiste. Au soir de sa vie, alors qu'elle s'apprête à regagner sa nuit, Betty voit la silhouette de son fils tendant les bras vers le ciel vespéral, image rayonnante du Fils de l'homme en gloire, confiant dans la promesse de l'aube à venir, d'un lendemain éternellement recommencé.

On ne trouvera plus ce message d'espoir dans les œuvres ultérieures, marquées par l'interdiction de s'évader en devenant autre, en devenant l'autre, fils ou amant. Annette Xanthner, l'héroïne de Die Tapetentür ${ }^{19}$, met au monde un enfant mort-né. Juste avant l'accouchement qui ne sera pas une délivrance, elle fait un long rêve. Par la porte dérobée de la chambre où elle repose, elle voit entrer un visiteur, le chien de ses jeunes années, Pluto(n), qui sur son dos lui fait franchir le seuil et l'emmène dans des profondeurs où elle retrouve la maison de son enfance. Mais celle-ci est vide. Les femmes sont allées à l'église. Le père est absent. Il est "parti pour toujours". Doublement prémonitoire, le rêve d'Annette lui annonce que l'enfant ne (re)viendra pas. Elle ne reverra son enfance ni dans les yeux de son propre enfant, ni dans le souvenir de sa mémoire. Le rêve referme définitivement la porte dérobée. Le futur est impossible, et impossible le retour au passé. Le chien Pluto(n), messager de « ce savoir qui s'est abattu sur elle avec une clarté glaciale», messager de la "connaissance» (Erkenntnis), est l'image antithétique de l'ange Gabriel. Il n'y aura pas de rédemption. Condamnée au présent, Annette devra vivre dans la déréliction, dans l'enfer d'un monde frelaté où les valeurs s'appellent «une auto, un compte en banque et un réfrigérateur » (D177, F145), où le bonheur n'est plus qu'un succédané, « la vie [...] toute entière un produit de remplacement» (ibid.). Elle a appris que sa "maladie était sa propre vie ", mais "elle refus(e) de guérir en devenant une personne étrangère qui n'aurait rien de commun avec elle » (D220 f., F179).

Les personnages de Marlen Haushofer ne sont pas suicidaires, au sens banal du terme. Ils respectent trop la créature pour se donner la mort. Même s'ils ne l'envisagent pas toujours avec une stoïque sérénité, ils finissent par s'en accommoder :

Dans ma jeunesse, j'étais assaillie parfois [...] par la peur de la mort. [...] Aujourd'hui

$[\ldots .$.$] je ne ressens aucune peur { }^{20}$.

L'obsession qui nourrit principalement leur névrose n'est donc pas de mourir, mais de vivre mal, de vivre le mal. Dans cette perspective, il convient moins de s'intéresser à la maladie de la mort qu'à son traitement, à la thérapie par l'autoanalyse réalisée dans les 
écrits. Marlen Haushofer ne parle-t-elle pas de ses œuvres comme d'enfants qu'elle rejette et qui dès lors ne la concernent plus, et de ses héroïnes comme d'autant de projections d'elle-même dont elle se détache au fur et à mesure de la cure que représente pour elle l'acte d'écrire ${ }^{21}$ ? Rechercher la guérison est donc moins lutter contre la mort que se réconcilier avec la vie. Marlen Haushofer est morte en 1970 d'un cancer des os, la maladie qui sans doute correspond le mieux à l'image d'un monde divisé, éclaté, chaotique, où les cellules anarchiquement prolifèrent sans tenir compte de leur fonction au sein d'un organisme. Il faut noter que la métaphore du cancer est déjà présente dans les textes du début. Ainsi dans Eine Handvoll Leben:

Elisabeth était devenue perméable et le monde entier l'envahissait. C'était comme si sa chair et ses os s'étaient liquéfiés [...] $]^{22}$.

Elle est particulièrement frappante dans un récit intitulé Die Ratte ${ }^{23}$. Mais surtout, l'anarchie du monde se reflète dans le dérèglement mental des héroïnes. Regula Venske et Irmela von der Lühe ont analysé ses deux aspects. La première, après avoir fait une légitime et sévère mise au point sur les critiques parues dans la presse, privilégie le lien entre le somatique et le social. Mais au lieu d'en apporter la preuve par les textes, elle fait un parallèle avec les œuvres de deux femmes écrivains de la nouvelle génération. LibuSa Monikovâ (Pavane für eine verstorbene Infantin, 1983), et Anne Duden, (Übergang, 1982), dont elle cite la formule: "Mein Körper ist mein Gedächtnis». Quant à la seconde, elle insiste sur les composantes psychiques, étudiant les notions de vide et de vacuité, de plein et de plénitude, et la nécessité d'ériger entre le monde et soi une barrière, un mur, une enveloppe qui, délimitant un espace intérieur et un espace extérieur, devient à la fois rempart et prison, défend contre les agressions du monde extérieur et signale l'enfermement autistique. Nous n'aborderons que brièvement cette problématique et renvoyons à l'étude d'Irmela von der Lühe ${ }^{24}$.

Les personnages de Marlen Haushofer ne trouvent pas leur place dans le monde. Elles se fabriquent une peau qui ne leur appartient pas vraiment, si bien qu'en définitive elles sont restées des enfants :

Prisonnière en moi vit une petite fille [...] qui aimerait sauter et danser comme les autres enfants. Mais on l'a enfermée comme on enferme toutes les petites filles qui ne peuvent pas grandir. Cela ne tient vraiment qu'à moi si je ne peux m'accommoder du présent ${ }^{25}$.

Une notation qui rappelle l'image de la petite Méta punie par ses parents et assise au fond de son tonneau, au début de Himmel, der nirgendwo endet ${ }^{26}$... Au centre du monde, l'enfant est pierre, arbre, animal; il est le tout avant de devenir la partie qui, se détachant progressivement, va acquérir, par l'expérience de l'altérité, une identité propre. Ici, la transition du collectif au «il» puis au «je» ne se réalise qu'imparfaitement, laissant subsister des séquelles traumatiques. Il va falloir explorer les replis de l'inconscient, tâche de toute une vie, qui trouve son aboutissement, son " dénouement », dans Die Mansarde.

Le thème en est le suivant : la narratrice, femme au foyer, occupe ses loisirs à réaliser, dans la mansarde de la maison familiale, des illustrations de livres pour une maison d'édition. Pendant une semaine, elle reçoit des lettres anonymes contenant les fragments d'un journal intime rédigé à l'époque où, atteinte d'une surdité psychosomatique, elle était isolée dans un chalet de montagne, gardée par un mystérieux personnage, le chasseur, X. La cause de son inhibition autistique ne sera révélée que par bribes. La sirène d'une voiture de pompiers ou de police, déclenchée 
intempestivement en pleine nuit, a brusquement fait revivre à la jeune femme son passé scotomisé, les sirènes des alertes aériennes et avec elles, enfoui au plus profond d'elle-même, son sentiment de culpabilité ${ }^{27}$. De "jardin secret", la mansarde va devenir prison, lieu où seront confrontés les fantasmes du refoulement jadis consignés dans les pages du journal (pensées et évocations des dessins), et les illustrations et réflexions de la femme mûre, passe-temps qui semblait n'être qu'exutoire à une vie trop rangée.

Ainsi la narratrice déclare-t-elle :

Je savais que là-haut, dans la mansarde, j'avais une tranche de passé à liquider. Je n'avais certes pas le sentiment qu'il s'agît du mien, mais le passé, quel qu'il soit, doit être liquidé. C'est une démarche douloureuse devant laquelle, toute ma vie, je me défile ${ }^{28}$.

Au début du roman est évoquée la monotonie de la vie de famille, avec ses visites dominicales au Musée de l'Arsenal, où Hubert, le mari, s'adonne à ses marottes, et où l'épouse retrouve, "derrière les vitrines" (D17, F20) une civilisation révolue, "un paisible royaume des morts" (D17, F21), essentiellement celui de l'Empire romain, auquel va sa prédilection. Au musée, elle se sent tellement « chez soi » (daheim) qu'elle en éprouve "quelque inquiétude" (ein bißchen unheimlich) (D19, F16), car cet univers englouti lui rappelle étrangement le monde qui l'environne :

Je ne suis pas [...] tout à fait sûre que la ville vive réellement, peut-être est-ce un lieu d'ébats pour des figurines qui ont le droit de s'agiter un peu avant qu'on ne les enferme dans des vitrines.

Elle retrouvera cette impression en lisant son journal de crise, à la date du 10 décembre :

Je rêve beaucoup en ce moment de villes en ruines et de paysages d'où les êtres humains ont disparu et où ne se dressent que des statues lépreuses ${ }^{29}$.

Ce rêve constituait aussi le point de départ du roman Die Wand: la paroi de verre y séparait l'héroïne d'un monde extérieur où bêtes et gens étaient pétrifiés, transformés en statues minérales - ultime aboutissement du destin de l'humanité moderne. Les " automates" s'y sont définitivement immobilisés, ces poupées sans cœur et sans cervelle que les femmes, en se contentant d'un ersatz de bonheur bourgeois, redoutent toujours de devenir ${ }^{30}$. L'unique salut est alors le repli dans la tanière, dans l'antre, la grotte (voir le récit Die Höhle ${ }^{31}$ ), la vallée ou l'alpage, le lieu clos plus ou moins vaste suivant l'acuité de la crise, mais qui renvoie toujours à la " maison » idéale de l'enfance heureuse. Au paroxysme de l'angoisse, la paroi de verre se rétrécit aux dimensions de l'enveloppe corporelle :

Aucun être humain ne m'a touchée depuis si longtemps que je craindrais d'éclater au moindre contact en mille petits cristaux de glace ${ }^{32}$.

La tension vient-elle à se relâcher, le refuge s'agrandit. Quand la femme sourde parviendra à descendre au village pour y faire ses courses, elle sera sur la voie de la guérison. Dans l'intervalle, ce sont les montagnes qui deviennent ses « deux gardiennes de prison », et aussi l'image de la condition du « couple » qu'elle forme avec X : isolée, rêvant seulement d'être reliée à l'autre par l'intermédiaire d'un aigle venant "parfois d'un coup d'aile apporter dans ses serres un peu de terre et de l'herbe d'une autre montagne ». Nous verrons, au-delà de la portée psychanalytique de cette notation, la résonance biblique qu'elle contient. Contentons-nous ici de citer la phrase qui suit immédiatement : 
J'aimerais bien être une montagne mais je n'en suis pas une, je ne suis que la courtilière qu'on humilie et qui s'étonne ${ }^{33}$.

Si la restructuration du psychisme passe par la projection sur des éléments, des minéraux ou des végétaux, une pierre, un arbre, un nuage, comme l'indiquent les premières pages de Die Wand ou de Die Mansarde, elle se fait essentiellement par le biais de l'identification à des animaux ${ }^{34}$. Dans le récit Der Wüstling ${ }^{35}$ (terme qui désigne un "débauché » mais aussi une "fauvette »!), le personnage principal, un homme cette fois, assimile toutes les femmes qu'il rencontre aux animaux les plus divers. Celle dont il tombe enfin amoureux est une femme-singe, la seule qu'il respecte vraiment et dont il attend qu'elle reconnaisse en lui le lion. Mais elle meurt virgo intacta, sans avoir accompli son destin, ne laissant derrière elle que son portrait en jeune guenon : tandis que l'homme accédera peut-être à l'humanité en s'accouplant enfin à une femme humaine, la jeune fille échoue à trouver son complément. Pour elle, l'espoir est rejeté dans un hypothétique au-delà :

L'espace d'un instant, il songea qu'un jour [...] la jeune femme-chimpanzé reviendrait chercher son bien [l'autoportrait]. Quand elle n'aurait plus à craindre le visage humain qui était le sien.

D'abord emblématiques, incarnant des traits de caractère, les figures d'animaux deviennent très vite les éléments d'un formidable bestiaire fantasmatique. Dans Die Wand, les frontières entre l'espèce humaine et les bêtes domestiques s'estompent déjà, bien que la symbolique reste encore sous-jacente. La vache-sœur incarne la part de fécondité et d'amour maternel. Le chien est l'image positive de la masculinité: raisonnable, rationnel, comme Peter, ou le Pluto(n) de Die Tapetentür, il a pour nom Luchs (Lynx), référence au fauve à l'œil perçant, et aussi au personnage mythologique de Lyncée, dont le regard pouvait " percer » les murailles les plus épaisses et voir ce qui se passait au Ciel et aux Enfers ${ }^{36}$. Quant à la chatte sans nom, anonyme comme la narratrice, elle est femme accomplie, domestique et sauvage, tour à tour câline et indépendante, aussi à l'aise à l'intérieur qu'à l'extérieur de la maison, être idéal dont on apprend par ailleurs qu'il est sans cesse menacé par la société des hommes. Adulte et mère, elle doit rester sur ses gardes pour protéger sa vie et sa progéniture :

Ne fais jamais confiance aux humains, chatte, ils ne veulent que te martyriser et ils tueront tous tes petits $!^{37}$

Toute jeune, elle est exposée aux convoitises, elle est la frêle adolescente du récit Menschenfresser ${ }^{38}$ :

La petite [...] bâilla, montrant un bout de palais rose et des dents blanches. C'était le bâillement d'une jeune chatte.

Assise en face de sa victime potentielle, dans le compartiment d'un train,

la dame bien charpentée [...] était maintenant très pâle, et ses taches de rousseur ressemblaient à de la boue qui l'eût éclaboussée. Une pauvre hyène tachetée. Et comme elle retroussait ses babines! On aurait dit qu'elle se ramassait avant de bondir.

Dans l'histoire intitulée Die Entfremdung, on s'en souvient, la culpabilité des femmes était symbolisée par les taches brunes maculant les robes blanches. Ces taches brunes réapparaissent donc sur le pelage de la hyène. Elles sont toujours ce qui salit, à la différence pourtant que la femme est désignée ici comme "une pauvre hyène ». La réprobation a fait place à la pitié. L'attirance des femmes - des mères - pour le fascisme en devient compréhensible et excusable. Il est dans leur nature saturnienne de vouloir dévorer leur progéniture. La dame du train a envie de manger l'adolescente parce 
qu'elle est mère et que toutes les mères phagocytent leurs enfants. Ainsi, le complexe de la Hyène, le complexe de Jocaste, abolit le complexe du $\mathrm{Coq}^{39}$. Passant du plan sociohistorique au plan socio-psychologique, le sentiment de culpabilité s'efface. Une étape est franchie dans le processus de libération par rapport à un passé obsédant, et qui empêche de vivre.

41 "Je ne suis qu'une courtilière qu'on humilie et qui s'étonne "... En s'identifiant à l'insecte "archétype de la laideur et de la méchanceté » (D93, F105), la narratrice va poursuivre la démarche libératoire qui lui permettra de conjurer «la folie qui s'est emparée de toute ma génération» (D95, F107). Un premier dessin lui présente une "vision de cauchemar ». Elle le déchire. Le suivant la satisfera pleinement. L'insecte détesté, objet de répulsion, suscite dès lors la pitié, et...

... sa couleur brune n'est pas laide, c'est la couleur de la terre (D93, F105).

D'exotique, l'animal est devenu familier. S'il est « nuisible », il ne s'attaque plus, comme la hyène, à ceux de son règne ; il est le prédateur des seules plantes... L'image supprime à la fois la référence au fascisme et celle à la mère dévoreuse. Cependant, trop radical, le remède va renvoyer à l'isolement qu'il convient de rompre :

Ce n'est pas gênant que les insectes aient l'air solitaires. Chacun d'eux est entouré d'une aura d'étrangeté. C'est ma propre étrangeté naturellement. Ils sont très bien ainsi, ce qui n'est pas le cas pour mes oiseaux. Je devrais pouvoir dessiner un oiseau qui n'aurait pas l'air solitaire. Je suis le seul obstacle à ma progression (D94, F106 $)^{40}$.

Serait-on revenu au point de départ? Pas exactement. Cette réflexion, extraite du journal à la date du 14 février, vient en réponse aux inquiétudes manifestées au début du roman par la femme retirée dans sa mansarde. Elle notait alors qu'elle avait jadis peint un étourneau qui paraissait en écouter un autre, perché dans le jardin voisin. Illusion sans doute, mais combien consolante. Or «ce petit dessin » avait « disparu pendant la guerre » (D20, F24, cf. D119, F133). Ainsi est défini le bonheur: ne plus se sentir seule, chanter à l'unisson avec les autres êtres l'hymne de la réconciliation et se dire :

Je suis un oiseau très heureux parce que je ne suis pas seul (D94, F106).

Plus tard, les oiseaux dessinés pendant la «maladie» tendent à faire de la femme sourde le centre rayonnant du monde, seule condition pour elle de communier dans l'amour universel. L'étourneau, l'hirondelle, " animal sociable en apparence mais qui ne pense qu'à son butin» (D20, F24), le pinson du Nord (Bergfink, "pinson des montagnes ", D118, F133) restent des esquisses décevantes jusqu'au jour où la pie semble marquer un aboutissement :

La pie est une image parfaite, dans son genre (D155, F174) ${ }^{41}$.

La pie fait côtoyer dans son plumage le blanc et le noir, le bien et le mal. Elle n'est ni bonne ni méchante, elle est. Réunissant les contraires, elle est l'image concrète de la relativité des valeurs telle que l'envisage le rationalisme masculin, défini dans le bref récit Eine sonderbare Liebesgeschichte (v. supra). La pie est donc "gage d'une existence paisible", mais en même temps, elle reste un oiseau solitaire, d'une perfection "glaciale» (D155, F174); elle représente une régression en regard de l'espérance fallacieuse mais salvatrice que laissait entrevoir l'étourneau d'« avant la guerre». Elle reste une construction de l'esprit qui ne satisfait pas le cœur et exclut le véritable amour.

Or la pie reprend, en négatif, l'image ambiguë sur laquelle se terminait Die Wand. Dans le roman de 1963, les corneilles perchées dans les arbres étaient des créatures soumises 
à la loi inexorable du temps apparemment "immatériel et immobile ", indifférent, omniprésent. La femme n'ayant plus de montre, leur apparition régulière à l'orée du bois lui permettait de mesurer l'écoulement de la durée, et elle se mettait à vivre «à l'heure des corneilles » (D204, F253). Avec « leur sang rouge sous le noir plumage », les corneilles rappelaient l'image des coqs du récit Entfremdung et signalaient la cruauté non seulement de la période hitlérienne, mais de toute histoire. Elles étaient l'allégorie du temps, fossoyeuses de la Mort et instrument de Saturne : ce dieu n'avait-il pas la corneille pour emblème et pour arme d'hast une faux dont la lame reproduisait la forme de son bec $^{42}$ ? Se familiariser avec ces oiseaux, c'était adopter leur "patience stoïque » (D196, F243), accepter « le bon et le mauvais » (ibid.), admettre, à l'issue d'une vie éphémère, l'inéluctabilité de la mort. Toutefois, seule la corneille albinos, monstre rejeté par ses congénères, permettait l'identification. Elle devenait l'image de la solitude assumée, de la nécessaire et sereine résignation à l'ordonnance du monde. En la rejoignant, l'exilée, l'étrangère, réalisait "son aspiration à entrer dans le silence blanc et sans douleur » (D121, F151) :

J'irai dans la clairière porter à manger à la corneille blanche. Elle m'attend déjà.

$(\text { Fin })^{43}$.

47 La pie bicolore, elle aussi de la famille des corvidés, "reprend», avons-nous dit, la corneille blanche pour la nier et la dépasser. Dans Die Mansarde, la femme sourde ne peut se contenter de ce "triste monstre ». Elle le range dans son tiroir et part à la recherche d'un symbole plus satisfaisant. Mais la sittelle qui sort de son pinceau se transforme en un être hybride, prend «des traits de reptile » qui ne lui plaisent pas (D161, F180 sq.). Elle a peur de la créature en gestation, qui pourrait bien être, elle aussi, un monstre (D160, Fl79), lui signalant qu'elle a cette fois basculé dans la folie, ou une mort sans sépulture. Or le même fantasme revient, des années plus tard, hanter la femme guérie de sa surdité autistique sous la forme d'un cauchemar éveillé, au moment où la coiffeuse place sur sa tête le casque du séchoir. Il s'agit alors d'une chrysalide de couleur gris-brun, pendant au bout d'un fil argenté et agitée de pulsations qui annoncent la naissance d'une créature énigmatique. La femme entrevoit "un miroitement bleu métallique» et ouvre les yeux pour échapper à cette vision angoissante (D137, F154 sq.). En effet, l'imago pourrait être une réincarnation de Fräulein Röder, reine coupable morte sans rédemption, ou encore, avec ses chatoyantes irisations, avatar du coq de Entfremdung, faisant revivre les forces du chaos, les artisans de la destruction et du néant. La vision se renouvelle, et l'enveloppe craquelée laisse apparaître « un œil pourpre ", « un mauvais œil » (D140, F157). Enfin, dans les dernières pages du livre, la narratrice descend à la cave, ce lieu où Pluto(n) avait entraîné Annette pour lui annoncer la perte de son enfance, avec l'intention « d'en finir une fois pour toutes avec la haine et la peur» (D196, F220). Et maintenant, « une créature aux yeux d'or " la regarde. La chrysalide a libéré un dragon, "un être qui a le droit de paraître solitaire ». Au fond de ses " yeux jaunes » se lit « une grande innocence et une grande ignorance ». La femme devenue dragon est arrivée au terme de son existence terrestre :

Mon être vidé n'était plus qu'une enveloppe sur le néant ${ }^{44}$.

Mais après sa descente vers les profondeurs chtoniennes, elle réalise son ascension céleste, bienheureuse elle remonte à la mansarde, les yeux fermés, « pour mieux voir les yeux jaunes et innocents du dragon » (D201, F226). 
À la fin du dernier roman, l'ultime métamorphose se réalise, l'ultime délivrance survient. Le dragon qui sort littéralement de la tête de la femme apporte la réconciliation définitive avec la mort, avec le monde, avec l'histoire, avec soi, en intégrant tous les symboles élaborés auparavant. La chrysalide de papier gris-brun, l'œil mauvais couleur de pourpre, l'irisation métallique cèdent la place aux teintes d'une innocence retrouvée qui se reflète dans un œil jaune, dans un œil d'or. Le fil d'argent, cordon ombilical qui retenait le nouvel être, a été coupé mais n'a pas disparu. L'argent et l'or ne se sont pas dissous. Saturne, dont les anciens alchimistes disaient qu'il avait le pouvoir de tuer les propriétés de ces deux métaux précieux, de les "dévorer ", a été vaincu ${ }^{45}$. Tournant le dos aux années de plomb, le dragon ouvre l'ère du paradis retrouvé. Figure du bonheur et de l'amour, il inverse aussi le symbole biblique présent dans l'Apocalypse de saint Jean. Le dragon biblique, "l'énorme Dragon, l'antique Serpent, le Diable ou le Satan » $(12,9)$ avait été envoyé par Dieu sur terre pour détruire Babylone-Rome coupable d'avoir décimé le peuple élu par une persécution sanglante. La femme de Die Mansarde, qui restait liée à un Empire romain réminiscence $d u \mathrm{III}^{\mathrm{e}}$ Reich, réussit à rompre les liens qui la rattachaient à lui et l'entravaient. Quant au refuge de montagne, où l'avait isolée sa " maladie », il était pour elle une prison, un enfer d'un an et demi, et non le « Désert » où « loin du Serpent, la Femme doit être nourrie un temps et des temps et la moitié d'un temps ${ }^{46}$ ». Si «les oiseaux vont se repaître de sa chair » $(19,21)$, ils ne sont plus pour elle «toutes sortes d'oiseaux impurs et dégoûtants" $(18,2)$. La corneille blanche est devenue colombe, l'esprit et l'époux qu'elle rejoint dans l'épilogue de Die Wand - renversement de l'Epilogue biblique où l'on peut lire: "L'esprit et l'épouse disent: "Viens" ». La courtilière, la corneille blanche et le dragon, reprenant la triade du Fils qui accomplit son destin sur terre, de l'Esprit Saint et du Père, marquent le rejet de la religion et de la tradition judéo-chrétiennes. Et plus que les deux autres symboles, le dragon divin, trinité dans l'Un, chimère, pur fantasme dans la Création, antithétique du monstre, est la Révélation. Vidée de sa substance corporelle, la Femme revendique son imaginaire. Elle n'a plus à rejeter ses enfants. Elle les reconnaît tous. Elle atteint à la plénitude par ses œuvres, témoignage de sa solidarité et de sa communion avec l'humanité. Elle réalise son idéal de "raison de l'amour et de la morale ${ }^{47}$ » en affirmant son statut d'artiste, sa vocation d'écrivain.

\section{NOTES}

1. - Die Mansarde, Roman, Ciaassen Verlag, Düsseldorf 1969, (2) 1984. Cité d'après Fischer TB, Frankfurt/M. 1987, D197. Ed. française : Dans la mansarde, trad. par Miguel Couffon, Actes Sud, Arles 1987, p. 221. D : édit. allemande référencée ; F : éd. française.

«Die Welt ist ein Irrgarten, in dem ich mich nie zurechtfinden werde, mit diesem Kopf, der nur für Bilder geschaffen ist und nicht für vernünftige Gedanken». 
2. - «Une agréable soirée à la maison ", in Begegnung mit dem Fremden (À la rencontre de l'étrange), Erzählungen Bd. 1, Ciaassen Verlag, Düsseldorf 1985. Ce vol. rassemble les 20 récits du recueil précédemment paru sous le titre Die Vergißmeinnichtquelle (La source des myosotis), et 27 récits jusqu'alors inédits.

3. - "Terrible fidélité », histoire-titre du second recueil de récits, Ciaassen Verlag, Düsseldorf 1968, augm. 1986. Contient Wir töten Stella, éd. fr. : Nous avons tué Stella, trad. par Yasmin Hoffman et Maryvonne Litaize, Actes Sud, Arles 1986.

4. - «Ich schreibe nie über etwas anderes als über eigene Erfahrungen. Alle meine Personen sind Teile von mir, sozusagen abgespaltene Persönlichkeiten, die ich recht gut kenne [...]. Ich bin der Ansicht, daß im weiteren Sinne alles,was ein Schriftsteller schreibt, autobiographisch ist», in «Marlen Haushofer oder die sanfte Gewalt, Ein Gespräch mit Elisabeth Pablé», April 1968, reprod. dans : Oder war da manchmal noch etwas anderes?, Texte zu Marlen Haushofer, Verlag Neue Kritik, Frankfurt/M. 1986.

5. - Regula Venske, dans son article «Vielleicht, daß ein sehr entferntes Auge eine geheime Schrift aus diesem Splitterwerk enträtseln könnte ..., zur Kritik der Rezeption Marlen Haushofers», in Oder, op. cit., p.60, parle de Kontinuität des Faschismus. Quant à Oskar Jan Tauschinski, Pami de toujours, il affirme sans pousser plus avant l'analyse qu'« une fois seulement dans l'œuvre de l'écrivain le démonique ne revêt pas les traits d'un individu mais s'incarne dans un événement historico-politique : le fascisme et la guerre ", citant le récit Der Staatsfeind («L'ennemi de l'Etat»), qui remonte à la toute première période de production de l'auteur, in Oder ..., op. cit., p. 167 sqq.

6. - «Entfremdung»: terme difficile à traduire, « Aliénation », c'est-à-dire «le fait de devenir étranger à soi-même ", in Begegnung mit dem Fremden, op. cit., p. 230 sqq.

«Und dann sah ich den großen Hahn [...]. Übermannsgroß, auf federbehosten schillernden Beinen, mit purpurrotem Kamm und schwarzem Schnabel stand er ganz ruhig und sah unsagbar fremd und prächtig aus» (234).

«Hier saß ich, eine Frau in zerbröckelnden Kleidern, die das letzte Kind unserer Welt in den zerfallenden Armen hielt» (237).

«Ich bin ja nicht einmal selbst ganz frei von Verrat und Treulosigkeit» (ibid.).

7. - « L'héritier », in Schreckliche Treue, op. cit., p. 109 sqq. Sur Melissa, Bienenkönigin, alte Frau, die jedes Jahr ihren Gemahl tötet, v. Robert von RankeGraves, Griechische Mythologie, Quellen und Deutung, 2 Bde, éd., Rororo TB 113/4, Reinbek bei Hamburg 1960, 7, 3.

8. - « Les enfants », in Schreckliche Treue, op. cit., p. 167 sqq.

9. - «Le temps ", in Schreckliche Treue, op. cit., p. 183 sqq. Cf. également Wir töten Stella, op. cit., D77, F41 : Das Ticken der Unrast.

10. - Dans la mansarde, op. cit., F50, D43f. ; F56, D49. - außerbürgerliche Ausschweifungen : D43, F49.

11. - «Eine sonderbare Liebesgeschichte» («Une singulière histoire d'amour »), in Schreckliche Treue, op. cit., p. 217 sqq. Cit. p. 220 : «Alles ist so verworren, ich müßte den Faden finden und ihn zurückspulen, aber ich finde den Anfang nicht, nur das Ende [...]». Cf. également Wir töten Stella, op. cit., p. 106 sq.

12. - «Gewisse Dinge», Die Mansarde, op. cit., D195, D219. Cf. D56, F64.

13. - «Die Geschichte vom Menschenmann", in Begegnung mit dem Frem den, op. cit.y p. 224 sqq. «Der Menschenmann»: «Le mâle humain », comme on dirait du mâle d'une espèce animale particulière.

14. - Die Wand, Roman, Sigbert Mohn Verlag, Gütersloh 1963. Rééd. : Ciaassen Verlag, Düsseldorf 1983, - Ullstein TB 30169, Frankfurt/M.-Berlin 1985, - Réf. dans : Editionen Pegasus, Klett Verlag, Stuttgart 1987. Ed. fr. : Le mur invisible, trad. par Liselotte Bodo et Jacqueline Chambon, Actes Sud, Arles 1985.

15. - Ibid., D40, F52. - Menschenfrau, littéralement : « femelle de l'homme », v. supra note 13. 
16. - Die Tapetentür, Roman, Paul Zsolnay Verlag, Wien 1957. Réf. dans l'éd. Knaur TB 8024, München s.d. - Ed. fr. : La porte dérobée, trad. par Jacqueline Chambon et Liselotte Bodo, Actes Sud, Arles 1988. Cit. suiv. : D95f., F79 sq.

17. - Eine sonderbare Liebesgeschichte, v. supra n. 11. «Ich weiß nicht, was ich dafür gäbe, wenn ich Peter endlich loswerden könnte. Keinen Groschen natürlich, ich will ihn gar nicht loswerden» (217). «Das Schlimme ist nur, daß jetzt, seit ich seine Gedanken denke, alle meine Handlungen mir für Augenblicke völlig unverständlich sind» (227). «Wir tun so, als liebten wir einander, aber wir wissen, daß wir keine andere Wahl haben. Auf der ganzen Welt gibt es keine Frau für Peter und keinen Mann für mich. Wir führen ein friedliches Leben» (230).

Les hérö̈nes en arrivent non seulement à penser comme l'homme, mais aussi à éprouver ses sentiments. Nous n'avons pas développé cet aspect de la perte d'identité. V. à ce propos les pertinentes remarques de Regula Venske (art. cité) sur la prétendue tendance lesbienne de M. Haushofer, en particulier d'après le roman Eine Handvoll Leben, op. cit., n. 18.

18. - Eine Handvoll Leben (« Une poignée de vie »), Roman, Paul Zsolnay Verlag, Wien, 1955. Réf. dans : Knaur TB 8023, Frankfurt/M. s.d. - «Ihr Verlangen nach dem Unerreichbaren verwandelte sich langsam in Traurigkeit» (141).

M. Haushofer elle-même, après un divorce prononcé aux torts de son mari, continua de vivre sous le même toit que lui et de lui servir d'assistante à son cabinet dentaire. "Elle était d'une bonté perverse", dit un témoin interrogé par Manuela Reichart, qui rapporte par ailleurs les propos du $\mathrm{D}^{\mathrm{r}}$ Manfred Haushofer, lequel aurait «pardonné » (à Marlen), avant leur mariage en 1941, un «faux-pas ", « comme on disait à l'époque », in Manuela Reichart, «Eine völlig normale Geschichte. Auf den Spuren von Marlen Haushofer - Ein Reise nach Österreich», Oder..., op. cit., p. 21 sqq. Cit. pp. 24 et 34 . Nous n'insisterons pas sur la connotation sexuelle du cheval (cf. par ex. la littérature américaine, Faulkner et Tandis que j'agonise, ou Car son McCullers et Reflets dans un œil d'or). Il y a au demeurant peu d'animaux « hippophalliques » dans l'œuvre de M. Haushofer.

19. - Die Tapetentür, op. cit., D 212 à 220, F 173 à 179.

20. - Die Mansarde, op. cit., D33f., F39. - «Als ich jung war, überfiel mich manchmal [...] Todesangst [..]. Jetzt spüre ich keine Angst».

21. - Marlen Haushofer confie : «Meine Bücher sind alle verstoßene Kinder. Mich interessiert nur der Vorgang des Schreibens. Die einzige Ausnahme ist der Roman "Himmel, der nirgendwo endet", eine Autobiographie meiner Kindheit. Auch dieses Buch lese ich nicht wieder, es genügt mir, in ihm ein Stück Vergangenheit eingefangen zu haben und manchmal daran zu denken», in M. Haushofer, «"Meine Bücher sind alle verstoßene Kinder", ein Gespräch mit Dora Dunkl», novembre 1969, Oder..., op. cit., p. 134-136, cit., p. 136. (« Mes œuvres sont toutes des enfants que j'ai rejetés. La seule chose qui m'intéresse, c'est l'écriture. Fait exception le roman "Un ciel qui ne confine nulle part", une autobiographie de mon enfance. Même ce livre, je ne le relis pas. Il me suffit de savoir que j'y ai enfermé une part de mon passé, et d'y songer de temps à autre »).

22. - «Elisabeth war durchlässig geworden und alle Welt durchströmte sie. Es war, als hätten sich ihr Fleisch und ihre Knochen in Flüssigkeit aufgelöst». Eine Handvoll Leben, op. cit., p. 165. Sur ce point, on notera cette réflexion révélatrice sur la mort et le suicide : «Es gibt keine Flucht. Der Tod ist nicht tödlich genug », ( «I n'y a pas de fuite possible. La mort n'est pas assez mortelle »), in Wir töten Stella, op. cit., D72, F32. V. également les propos de O.J. Tauschinski rapportés par Manuela Reichart et selon lesquels, de l'avis d'un médecin, le cancer (son cancer) était une maladie voulue, voulue inconsciemment, une sorte de suicide inavoué. (Art. cité p. 27).

23. - «Die Ratte», (« Le rat »), in Schreckliche Treue, op. cit., p. 117 sqq.

24. - Regula Venske, art. cité. Irmela von der Lühe, «Erzählte Räume - leere Welt. Zu den Romanen Marlen Haushofers», in Oder..., op. cit., p. 73 sqq. Sur cet aspect et les notions de Begrenzung et Entgrenzung, v. les analyses de DeleuzeGuattari, en part, dans L'anti-Edipe, qui définissent les concepts de « territoire », « exterritorialisation » et « reterritorialisation ». 
25. - Die Mansarde, op. cit., D183, F205sq. - «Eingesperrt lebt in mir ein kleines Mädchen, das [...] herumtanzen möchte wie alle anderen Kinder. Aber man hat es eingesperrt, und das passiert allen kleinen Mädchen, die nicht aufhören kleine Mädchen zu sein. Es liegt wirklich nur an mir, wenn ich mich mit der Gegenwart nicht abfinden kann».

26. - Himmel, der nirgendwo endet ..., Roman, Sigbert Mohn Verlag, Gütersloh 1966 ; rééd. Ciaassen Verlag, Düsseldorf 1984; Fischer TB 5997, Frankfurt/M 1986. - Sur les phénomènes et objets transitionnels, évoqués par la narratrice de Die Mansarde à propos de ses enfants (op. cit., D44 sq., F51 sq.), v. la déf. de l'« aire intermédiaire d'existence » et l'ensemble des analyses de D.W. Winnicott.

27. - Die Mansarde, op. cit., surtout D64 et 185, F73 et 207 sq.

28. - Ibid., D43 sq, F50. - «Ich wußte, daß ich dort oben in der Mansarde ein Stück Vergangenheit zu liquidieren hatte. Zwar hatte ich nicht das Gefühl, es handelte sich nicht um meine eigene Vergangenheit, aber jede Vergangenheit gehört ja liquidiert. Das ist ein schmerzlicher Prozeß, vor dem ich mich mein ganzes Leben lang drücke». V. également D49, F56.

29. - Ibid., D17, F21 et D89, F101. - «Ich bin auch nicht ganz sicher, ob die Stadt wirklich lebte oder ob sie nicht ein Tummelplatz ist für Figuren, die noch ein bißchen zappeln dürfen, ehe man sie in Glasvitrinen sperren würde. - Ich träume jetzt viel von zerfallenen Städten und von Landschaften, in denen es keine Menschen mehr gibt, nur verwitterte Statuen».

30. - Image obsessionnelle chez M.H., dans de nombreuses œuvres. Pour Die Mansarde, v. surtout D.17, F21 ; D45, F52 (« Automate ») ; D164, F184. - Wir töten Stella, op. cit., D70, F30.

31. - «Die Höhle» («La grotte»), in Begegnung mit dem Fremden, op. cit., p. 260 sqq. V. ce que la narratrice de Die Mansarde dit de son foyer : « ... je sus que je n'étais pas ici chez moi. Mais si je ne dois pas me sentir chez moi, je préfère que ce soit ici plutôt qu'en tout autre lieu. En avoir conscience, c'est déjà un grand bonheur » (F108, D96).

32. - Die Mansarde, op. cit., D87, F98. «So lange hat mich kein Mensch berührt, daß ich fürchte, ich könnte bei der ersten Berührung zerspringen, in tausend kleine Eiskristalle». L'idée n'est pas nouvelle, si l'on songe à Descartes parlant de ces êtres qui « s'imaginent être des cruches ou avoir un corps de verre » (Première méditation, éd. Gallimard, Pléiade, Paris, 1953, p. 268).

33. - Die Mansarde, op. cit., Dl 18, F132. «Schön wäre es, ein Berg zu sein, aber ich bin kein Berg, ich bin immer nur die gekränkte und erstaunte Maulwurfsgrille».

34. - Les images fantasmatiques des romans de M.H. évoquent des phénomènes transitionnels correspondant à des constantes universelles. Si Die Mansarde s'ouvre sur l'image d'un arbre dont les racines plongent dans le sol et les branches saisissent le ciel, c'est plus à une "psychanalyse par les animaux" qu'à une "psychanalyse par les plantes" que se livre l'auteur: "Je n'ai toujours dessiné que des insectes, des poissons, des reptiles et des oiseaux. Je ne me suis jamais attaquée ni aux mammifères ni aux humains. Je saurais dessiner les fleurs, mais elles ne m'ont jamais beaucoup attirée » (F23, D19 sq.). On consultera avec profit l'excellent ouvrage de Tobie Nathan, Psychanalyse et copulation des insectes, Ed. La pensée sauvage, Grenoble, 1979/1983 (2). Il y est dit : «Un homme peut assurément produire une grande quantité de fantasmes, mais pas une infinité; même très étendue, la série est limitée»(p.13). T. Nathan précise qu'il y a correspondance exacte entre les fantasmes sexuels (inconscients) et les différents modes de copulation chez les animaux inférieurs, notamment les invertébrés, mais aussi les poissons et les reptiles, étant donné que c'est chez ces espèces qu'on rencontre les modes de copulation les plus variés. Un psychanalyste pourrait ainsi, en lisant romans et récits de M. Haushofer, dresser son portrait psychique et dégager une dominante schizophrénique et des caractères secondaires, hystériques et mégalomaniaques, par ex.

35. - «Der Wüstling», in Schreckliche Treue, op. cit., p. 197 sqq. On pourrait ajouter à la série des femmes-animaux de ce récit les «femmes-abeilles » d'Hubert, dans Die Mansarde (42, F49), où l'on rencontre aussi une femme-furet (maléfique). - « ...einen Augenblick lang ging ihm durch den Kopf, daß irgendwann nach seinem Tod das kleine Schimpansenmädchen zurückkommen und 
sein Eigentum abholen werde. Dann, wenn es sich nicht mehr vor seinem Menschengesicht fürchten mußte» (205).

36. - V. Dictionnaire encyclopédique Quiltet, Paris, 1953; Robert von RankeGraves, Griechische Mythologie, op. cit., I, 60 et 74. M. Haushofer avoue sa " grand » peur des chiens, autrement dit de l'homme !: «Für eine vergeßliche Zwillingsschwester, Nachruf zu Lebzeiten», in Oder..., op. cit., p. 123.

37. - Die Mansarde, op. cit., D124, Fl 39. «Vertrau keinem Menschen, Katze, sie wollen dich nur quälen und bringen alle deine Kinder um». Hans Weigel rapporte que M. Haushofer lui remit le manuscrit de Die Wand en disant que c'était «l'histoire d'une chatte» (Die Geschichte von einerKatz), ce qui n'était pas simple modestie de sa part, in Oder..., op. cit., p. 29 sq. À la fin de Die Mansarde, Malina est un matou sympathique...

38. - «Menschenfresser» («Les ogres »), in Schreckliche Treue, op. cit., p. 124 sqq. «Die Kleine [...] gähnte, ein Stück rosa Gaumen und weiße Zähne zeigend. Es war das Gähnen einer jungen Katze [...]. [...] Die knochige Dame [...] war jetzt sehr bleich, und die Sommersprossen sahen aus wie Schmutzspritzer. Eine arme gefleckte Hyäne. Und wie sie die Lippe hochgezogen hatte, als duckte sie sich gerade zum Sprung» (129). Nombreuses réf. au thème anthropophagique. V. par ex. Wir töten Stella, D71-73, F31 sq. : Fleisch comme viande et chair humaine. - Die Mansarde, D14, F18...

39. - V. l'ouvrage de Christiane Olivier, Les enfants de Jocaste. L'empreinte de la mère, Gonthier/ Denoèl, Paris, 1980. La couverture du livre reproduit un détail du tableau symboliste de F. Khnopff, «L'art, les caresses ou le Sphinx », représentant un jeune adolescent à visage androgyne et une femme-sphinx à tête humaine et corps de hyène tachetée (Musées royaux des beaux arts, Bruxelles).

40. - Die Mansarde, op. cit., «Das Urbild des Häßlichen und Bösen». - «Die Verrücktheit, die meine ganze Generation befallen hat [...]». - «Ihr Braun ist nicht häßlich, sondern die Farbe der Erde». «Bei den Insekten stört es nicht, daß sie so einsam aussehen. Jedes von ihnen ist umgeben von einer Aura der Fremdheit, meiner eigenen Fremdheit natürlich. Sie sind ganz in Ordnung so, nicht aber meine Vögel. Ich weiß, es müßte möglich sein, einen Vogel zu zeichnen, der nicht einsam ist; nur ich stehe mir dabei im Wege».

41. - Ibid., «ich bin ein sehr glücklicher Vogel, weil ich nicht allein bin». - «Die Elster ist in ihrer Art ein vollkommenes Bild».

42. - R.v. Ranke-Graves, Griechische Mythologie, op. cit., 1,7,2 : «Kronos (war) ein Krähentitan, die geschlachteten Opfer (wurden) an heilige Krähen verfüttert». - 1,7,1: «Die Hippe, die Saturn, das lateinische Äquivalent des Kronos, trug, war wie ein Krähenschnabel geformt». Au même endroit, il est signalé que Rhea s'apparenterait à la déesse trinitaire Dione ou Diane, dont les emblèmes sont le chêne et la colombe, cette dernière étant aussi un animal « aphrodisiaque » $(1,11,2)$. Ainsi, la corneille blanche serait à la fois Eros et Thanatos (v. n.43).

43. - Die Wand, op. cit., p. 226 : « ...werde ich auf die Lichtung gehen und die weiße Krähe füttern. Sie wartet schon auf mich ».

44. - Die Mansarde, op. cit., D197 sq., F222, «[...] ich war ausgehöhlt, eine Hülle über dem Nichts».

45. - V. par ex. art. saturne et saturnisme in : Dictionnaire encyclopédique Quiltet, op. cit.

46. - Apocalypse de saint Jean, 12,14. Signalons que la romancière démiurge fait commencer son œuvre un dimanche, inversant ainsi l'ordre chronologique de la Genèse ; que la narration s'étend sur une semaine plus un jour, puisqu'elle se clôt aussi un dimanche, jour du « repos » et de la «révélation» ultime. De même, la narratrice du Mur invisible commence son récit au bout de deux ans et demi de réclusion dans ses montagnes.

47. - «Die Vernunft der Liebe und der Moral», cité par Regula Venske, art. cité, in Oder..., op. cit., p. 51. 


\section{RÉSUMÉS}

Les récits et romans de Marlen Haushofer cachent sous la banalité apparente d'une réalité quotidienne un univers d'une saisissante complexité, qui plonge dans l'obscurité du mythe et de l'inconscient. Hantée par la solitude, le sentiment d'une faute, le déchirement tragique, le besoin de connaissance et d'amour, la psyché de la femme reflète des images composant un fantastique bestiaire onirique où se côtoient le coq et la hyène, l'abeille et la courtilière, la corneille et la pie, le veau, la vache et le dragon.

Hinter dem Schein der plattesten Alltäglichkeit bergen Marlen Haushofers Erzählungen und Romane eine bis in die finsteren Tiefen des Mythos und des Unbewußten hinabführende Welt. An Einsamkeit, Schuldgefühl, tragischer Zerrissenheit leidend, sehnt sich die Frau nach Erkenntnis und Liebe. Ihre Psyche spiegelt Bilder eines fantastischen Traumbestiariums wider, in dem Hahn und Luchs, Maulwurfsgrille und Biene und nicht zuletzt Elster, Krähe, Star und Drache einander begegnen.

\section{AUTEUR}

\section{PATRICK CHARBONNEAU}

Université de Lille III 